Classification

Physics Abstracts

$3620 \mathrm{r}-6125 \mathrm{H}$

\title{
Precipitation of Polyelectrolytes in the Presence of Multivalent Salts
}

\author{
J. Wittmer, A. Johner and J.F. Joanny \\ Institut Charles Sadron, 6 rue Boussingault, 67083 Strasbourg Cedex, France
}

(Received 24 October 1994, received in final form 13 December 1994, accepted 16 December 1994)

\begin{abstract}
We present a theory of the complexation of polyanions by divalent cations. The formation of both mono-and dicomplexes is possible. Monocomplexation locally inverts the charge of the polyelectrolyte and transforms it into a polyampholyte. The formation of a dicomplex creates a bridge between charged monomers. The complexation constants for mono and dicomplexation strongly decrease at high ionic strength. If the complexation constants are large enough, the polyelectrolyte precipitates at moderate salt concentrations. The precipitation is driven either by a Debye-Hückel polarisation energy as in polyampholytes (for almost pure monocomplexation) or by the bridging attraction induced by the dicomplexes. The equilibrium constant for the transformation of two monocomplexes into a dicomplex (at vanishing ionic strength) is a key parameter : - when monocomplexation is favored, the polymer is redissolved by strong charge inversion at moderate salt concentrations. Two successive polymer precipitations and solubilisations can be observed as the divalent salt concentration is increased. - when dicomplexation is favored, the precipitation at moderate salt concentration is followed by a redissolution at high ionic strength where complexation becomes unefficient. Only one precipitation is expected.
\end{abstract}

\section{Introduction}

Polymer solutions in organic solvents have been studied extensively over the last decades both experimentally and theoretically [1]. Much applied experimental work has however focused on water-soluble polymers which are of considerable practical interest.

The polymer backbones are in general modified hydrocarbon chains which are insoluble in water. A collapse of the solution can be driven by the short-range attractions between monomers. The most important class of water-soluble polymers is charged polymers or polyelectrolytes. The long-range repulsive electrostatic interactions between monomers [2] with the same electrostatic charge dominates over the short-range attractions [3] and allows the solubilization.

In the presence of a monovalent salt, the electrostatic interactions are screened over a range 
$\kappa^{-1}$, the Debye-Hückel screening length, given by $\kappa^{2}=8 \pi l_{\mathrm{B}} \phi_{\mathrm{S}}$ where $l_{\mathrm{B}}=\frac{e^{2}}{4 \pi \varepsilon k_{\mathrm{B}} T}$ is the Bjerrum length (approximately equal to $0.7 \mathrm{~nm}$ in water) and $\phi_{\mathrm{S}}$ is the concentration of monovalent salt. Only upon massive addition of monovalent salt does the polyelectrolyte usually precipitate [4].

In marked contrast, polyelectrolytes precipitate often quite easily upon addition of multivalent salt [5]: a typical example is the addition of a calcium salt to a polycarboxylate solution. A large excess of salt can in certain cases resolubilize the polymer $[6,7]$. This effect is rather general and of considerable practical importance. It is to be avoided whenever polyelectrolytes are used as thickeners, e.g., in oil recovery. On the other hand, the effect may be used to eliminate heavy metals, e.g., in the treatment of waste waters. The precipitation induced by multivalent salts is also effective at interfaces and the trapping of calcium ions by natural polyelectrolytes could explain the enhanced stability of teeth against acidic solutions [8].

Many experimental sets of data on polyanion/multivalent cation systems have been reported in the literature. Of major interest to us are the so called type-L systems [9] where the polyelectrolyte precipitates at low ionic strength. Along the lower precipitation line in a salt versus polymer phase diagram, the polymer and salt concentrations are proportional, whereas the upper redissolution line (not always present) corresponds to a fairly constant salt concentration Sometimes there is an additional gelation line in the diagram in the high polymer/low salt region [10] corresponding to the formation of a physical gel where the calcium ions act as crosslinks. Beyond these rather general features there exists a rich variety of diagram shapes.

Two types of mechanisms have been proposed to explain the interactions between small ions and polyelectrolytes: specific interactions leading to complex formation and Manning ion condensation $[11,12]$, driven by electrostatic forces. The complexation is a monomer property whereas the condensation requires chain connectivity. A similar complexation mechanism has been studied in detail for neutral polymers by Pezron and Leibler [13] or for the formation of water/polymer complexes by Tanaka [14].

In the following, we essentially consider weak polyelectrolyte solutions where Manning condensation plays no role. We also restrict ourselves to the weak coupling limit where $\kappa l_{\mathrm{B}}<1$. The specific interactions between a cation and the polyelectrolyte are described phenomenologically by effective binding energies that mimic the rather complicated processes involving ion hydration. We discuss only divalent cations $\left(\mathrm{Ca}^{2+}\right.$ ) and polyanıons (polycarboxylates, polysulfonates) that are the most relevant experimental systems. Two binding energies are needed, $\varepsilon_{1}$ for the formation of a monocomplex and $\varepsilon_{2}$ for the formation of a dicomplex.

The next section discusses the spinodal decomposition that leads to the macroscopic phase separation of the solution into two phases. In Section 3 the possibility of mesophase formation, prior to the macroscopic separation, is investigated.

\section{Macroscopic Phase Separation}

We consider a semi-dilute solution of a weak polyanion with a fraction $f$ of charged monomers at a concentration $\phi$ in the presence of a calcium salt (say chloride) at a concentration $\phi_{S}$. The interaction between the calcium ions and the polyanions leads to the formation of i) monocomplexes and ii) dicomplexes.

2.1. Qualitative Discussion. - i) A monocomplex is formed when a calcium ion binds onto one negative site of a polyelectrolyte where the electrostatic charge is locally inverted. The polyelectrolyte behaves then as a polyampholyte. Polyampholytes are known to precipitate when their net charge approximatively vanishes; the precipitation is due to the attraction 
between charges of opposite signs. A further adsorption of calcium ions leads to a charge inversion and therefore to a redissolution of the polymer. The entropy of the small counterions tends to stabilize the solution against phase separation. When only monocomplexes are formed, we expect a rather narrow insoluble region in the $\phi_{S}$ versus $f \phi$ diagram. In the limit of strong complexation where most calcium ions form monocomplexes, the precipitation is located around the line $\phi_{\mathrm{S}}=f \phi / 2$ of the phase diagram where the polymer is neutral.

ii) A dicomplex is formed by the binding of a calcium ion to two negative sites on the polyelectrolyte. The complexation of two monomers by a calcium ion is equivalent to an effective attraction. This attraction can be qualitatively described in terms of a negative second virial coefficient. The solution precipitates when the attractive interaction induced by the complexation is balanced by the osmotic pressure of the small ions.

Upon addition of a large excess of calcium ions, the ionic strength of the solution increases and calcium binding becomes less favorable: the binding constant $\mathbf{K}_{2}$ decreases with the ionic strength. A redissolution is expected when the chemical potential of a free charge $\approx-\kappa l_{\mathrm{B}}$ is comparable to the binding energy. This occurs at very high ionic strength where our results are only qualitative. In contrast to the case where only monocomplexes form, the precipitation of the solution can occur over several orders of magnitude in calcium concentration. The redissolution line can be suppressed if the binding energy is too large.

\subsection{Free Energy and Spinodal Line}

The solution contains:

- the polyelectrolyte at an average concentration $\phi$ contributing $-f \phi$ to the average charge concentration $\rho$

- the monovalent counterions, say sodium ions, at an average concentration $\phi_{\mathrm{Na}}=f \phi$

- the calcium ions at average concentration $\phi_{S}$ and their monovalent counterions, say chloride ions, at an average concentration $2 \phi_{\mathrm{S}}$.

A concentration $\phi_{1}$ of the calcium ions are bound and form monocomplexes while a concentration $\phi_{2}$ are bound and form dicomplexes, the density of free calcium ions is $\phi_{\mathrm{f}}=\phi_{\mathrm{S}}-\phi_{1}-\phi_{2}$.

We study only semi-dilute solutions and, in a first approximation, we consider the chain length as infinite. We also consider a $\theta$-solvent where the second virial coefficient $v$ between neutral monomers due to non-electrostatic interactions vanishes. Quite generally, $v$ is negative in water but the corresponding phase separation at high ionic strength is not specific to multivalent ions and has already been studied in details [4]. We include here a contribution of the positive third virial coefficient that stabilizes the solution.

We finally retain the following free energy:

$$
\begin{gathered}
\frac{F}{k_{\mathrm{B}} T}=-f \phi \log (f \phi)+\phi_{1} \log \left(\phi_{1}\right)+\phi_{2} \log \left(2 \phi_{2}\right)+\left(f \phi-\phi_{1}-2 \phi_{2}\right) \log \left(f \phi-\phi_{1}-2 \phi_{2}\right) \\
+\phi_{\mathrm{Cl}} \log \left(\phi_{\mathrm{Cl}}\right)+\phi_{\mathrm{Na}} \log \left(\phi_{\mathrm{Na}}\right)+\left(\phi_{\mathrm{S}}-\phi_{1}-\phi_{2}\right) \log \left(\phi_{\mathrm{S}}-\phi_{1}-\phi_{2}\right) \\
-\varepsilon_{1} \phi_{1}-\varepsilon_{2} \phi_{2} \\
+\frac{w^{2}}{6} \phi^{3} \\
-\frac{1}{12 \pi}\left[4 \pi l_{\mathrm{B}}\left(f \phi+4 \phi_{\mathrm{s}}+\phi_{\mathrm{Cl}}+\phi_{\mathrm{Na}}-4 \phi_{1}-6 \phi_{2}\right)\right]^{3 / 2}
\end{gathered}
$$

The terms of the first line account for the entropy of mixing of the charged monomers and of the complexes along the polymer. They are obtained from the number of complexions of $n$ sites 
(the negatively charged sites of the polymer) comprising $n_{1}$ labeled sites (involved in monocomplexes) and $n_{2}$ labeled pairs (involved in dicomplexes), $\Omega=\frac{n !}{2^{n_{2}} n_{1} ! n_{2} !\left(n-n_{1}-2 n_{2}\right) !}$. This estimate of the entropy is rather rough and ignores in particular the conformational entropy of the polymer due to loop formation.

The terms of the second line accounts for the entropy of the small ions. The polymer having a very large molecular weight, we neglect the translational entropy of the chains; we thus take the limit of infinite molecular weight. The terms in the third line are the effective binding free energies, they include any contribution linear in concentration due to complex formation such as the entropic contribution associated with the formation of loops to linear order. The term in the fourth line is the third virial contribution.

The last term is the electrostatic polarization energy of the solution. As in our previous study on polyampholytes where the charges are randomly distributed along the chain, the polarization energy is the same as that of a simple electrolyte solution where all the charges contribute to the screening: the screening length is given by $\kappa^{2}=4 \pi l_{\mathrm{B}}\left(f \phi+4 \phi_{\mathrm{S}}+\phi_{\mathrm{Cl}}+\phi_{\mathrm{Na}}-4 \phi_{1}-6 \phi_{2}\right)$. This polarization energy can be obtained by summing the free energy of the fluctuations of the charge density at a given wavevector over all wave vectors.

The average concentrations of monocomplexes $\phi_{1}$ and dicomplexes $\phi_{2}$ minimize the free energy (Eq. (1)). The minimization leeds to the classical chemical equilibrium laws:

$$
\begin{aligned}
\mathbf{K}_{1} & =\frac{\phi_{1}}{\left(f \phi-\phi_{1}-2 \phi_{2}\right)\left(\phi_{S}-\phi_{1}-\phi_{2}\right)} \\
\mathbf{K}_{2} & =\frac{\phi_{2}}{\left(f \phi-\phi_{1}-2 \phi_{2}\right)^{2}\left(\phi_{S}-\phi_{1}-\phi_{2}\right)}
\end{aligned}
$$

where $\mathbf{K}_{1}=K_{1} \cdot \exp \left(-2 \kappa l_{\mathbf{B}}\right)$ and $\mathbf{K}_{2}=K_{2} \cdot \exp \left(-3 \kappa l_{\mathbf{B}}\right)$ are the equilibrium constants. The bare equilibrium constants are defined as $K_{1}=\exp \left(\varepsilon_{1}+1\right)$ and $2 K_{2}=\exp \left(\varepsilon_{2}+2\right)$. In general, the chemical binding equilibrium constants explicitly depend on the potential $\psi$ at the surface of the polyion. We consider here only weakly charged polyelectrolytes for which this potential is small. The electrostatic contribution to the binding constants is essentially due to the polarization effect of the ionic solution. At vanishing ionic strength the equilibrium constants are equal to their bare values $K_{1}$ and $K_{2}$. At high ionic strength the equilibrium constants decrease exponentially. At high ionic strength our approach is only qualitative. The chemical potential of a charge $z e$ in the solution is $-z^{2} \kappa l_{\mathrm{B}} / 2$. The argument of the exponential in $\mathbf{K}_{1}$ corresponds to the suppression of a divalent calcium charge while that in $\mathbf{K}_{2}$ corresponds to the suppression of the calcium charge and two monomer charges.

The stability of the homogeneous solution requires the convexity of the free energy (Eq. (1)) with the equilibrium monocomplex and dicomplex concentrations given by the chemical equilibrium laws (Eq. (2)). We now discuss this stability by determining the spinodal line. The spinodal line where the homogeneous solution becomes unstable is the line of the $f \phi-\phi_{\mathrm{S}}$ phase diagram where the determinant $S$ of the second derivatives of the free energy vanishes:

$$
S=\left\|\left.\frac{\partial^{2} F}{\partial \phi_{\imath}^{2}}\right|_{\mathrm{eq}}\right\|=0
$$

The variables $\phi_{\mathrm{i}}$ are a set of independently fluctuating concentrations. We discuss here macroscopic phase separation, we thus look for an instability of the solution at a vanishing wave vector. The global electroneutrality reduces the number of independent concentrations by one. 
We retain, as independent variables, the polymer charge concentration $x$, the complex concentrations $\phi_{1}, \phi_{2}$, the total divalent salt concentration $\phi_{\mathrm{S}}$ and the counterion concentration $\phi_{\mathrm{Na}}$.

To proceed, we first consider the case where only monocomplexes form $\left(\phi_{2}=0\right)$. We then turn to the case where the calcium ions only form dicomplexes $\left(\phi_{1}=0\right)$. Finally, we discuss the more general case where both monocomplexation and dicomplexation occur.

2.3. Formation of Monocomplexes. - The determinant $S$ of the second derivatives of the free energy contains the following sets of terms:

- terms coming only from the entropy $S_{\mathrm{e}}$

- terms arising from the charge fluctuations (polarisation) $S_{\mathrm{p}}$

- terms arising from the short-range interactions (third virial) $S_{\mathrm{w}}$

- a mixed interaction contribution $S_{\mathrm{pw}}$.

The entropic contribution reads:

$$
S_{\mathrm{e}}=\frac{\left(x-2 \phi_{1}\right)^{2}}{2 \phi_{\mathrm{S}} \phi_{1} x^{2}\left(\phi_{\mathrm{s}}-\phi_{1}\right)\left(x-\phi_{1}\right)}
$$

It is always positive and thus the entropic terms in the free energy stabilize the solution. When $\phi_{1}=x / 2$, the polymer becomes neutral. A fluctuation in the polymer concentration can then preserve a homogeneous distribution of the small ions, and this entropic contribution vanishes. Note that we have ignored here the translational entropy of the chains proportional to $1 / N$. The electrostatic polarization contribution $S_{\mathrm{p}}$ is negative and thus destabilizing. In general, it is smaller than the entropic contribution $S_{\mathrm{e}}$ by a factor of order $l_{\mathrm{B}}^{3 / 2} c^{1 / 2}$ where $c$ is a typical concentration in the solution.

$$
S_{\mathrm{p}}=4 \pi l_{\mathrm{B}}^{2} \kappa^{-1} \frac{\left[-18 \phi_{\mathrm{S}} \phi_{1}^{2}+16 \phi_{1}^{3}+24 \phi_{\mathrm{S}} \phi_{1} x-24 \phi_{1}^{2} x-9 \phi_{\mathrm{S}} x^{2}+10 \phi_{1} x^{2}-x^{3}\right]}{2 \phi_{\mathrm{S}} \phi_{1} x^{2}\left(\phi_{\mathrm{S}}-\phi_{1}\right)\left(x-\phi_{1}\right)}
$$

The order of magnitude of the third virial contribution is $w^{2} f^{-3} c^{2}$ times that of the entropic term $S_{\mathrm{e}}$. The third virial interaction is therefore only relevant at fairly high concentrations where it contributes to the stabilization of the solution.

$$
S_{\mathrm{w}}=w^{2} f^{-3} \frac{x^{4}+6 \phi_{\mathrm{S}} x^{3}-4 \phi_{1}^{2} x^{2}}{2 \phi_{\mathrm{S}} \phi_{1} x^{2}\left(\phi_{\mathrm{S}}-\phi_{1}\right)\left(x-\phi_{1}\right)}
$$

The mixed interaction term $S_{\mathrm{pw}}$ is of order $l_{\mathrm{B}}^{3 / 2} w^{2} f^{-3} c^{5 / 2}$ times $S_{\mathrm{e}}$; it is only relevant at high concentration.

$$
S_{\mathrm{pw}}=4 \pi l_{\mathrm{b}} \kappa^{-1} w^{2} \frac{18 \phi_{\mathrm{S}} \phi_{1}^{2} x^{2}-16 \phi_{1}^{3} x^{2}-18 \phi_{\mathrm{S}}^{2} x^{3}+17 \phi_{1}^{2} x^{3}-3 \phi_{\mathrm{S}} x^{4}}{2 \phi_{\mathrm{S}} \phi_{1} x^{2}\left(\phi_{\mathrm{S}}-\phi_{1}\right)\left(x-\phi_{1}\right)}
$$

From these estimates of the various terms, we conclude that the stabilizing entropic contribution $S_{\mathrm{e}}$ dominates almost everywhere in the $x, \phi_{\mathrm{S}}$ plane, provided that the solution remains fairly dilute. The precipitation induced by polarization effects $\left(S_{\mathrm{p}}\right)$ can only occur close to the line of polymer neutrality where the entropic terms vanish. This is consistent with what is expected for polyampholyte chains that collapse only when they are approximately neutral. 


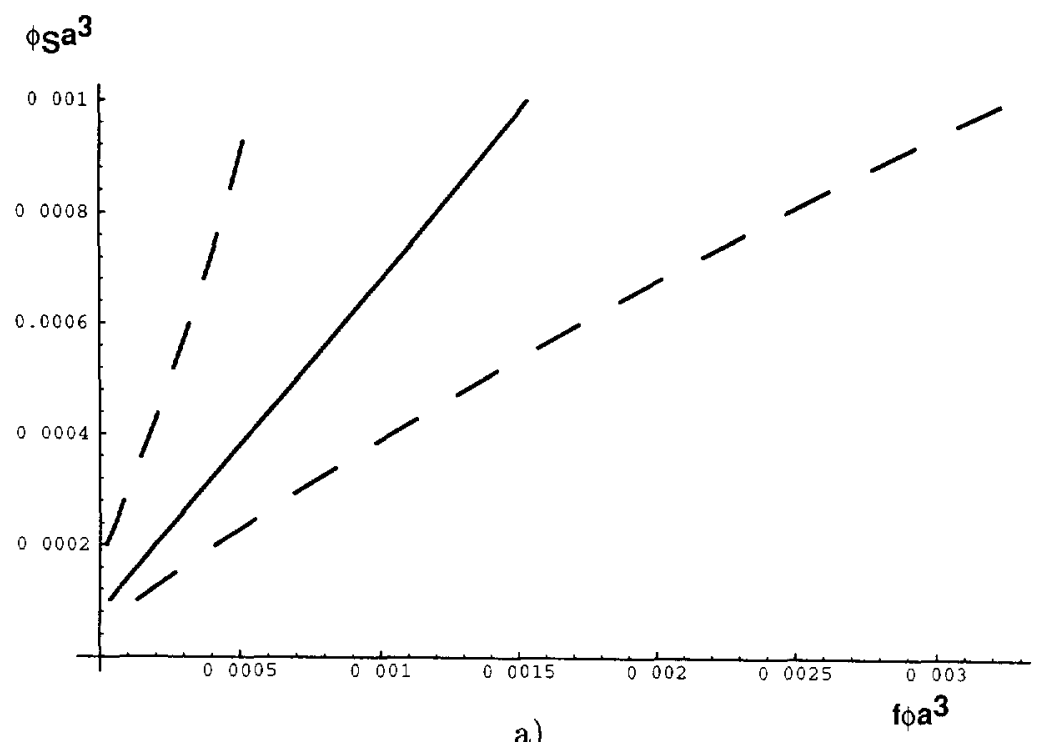

a)

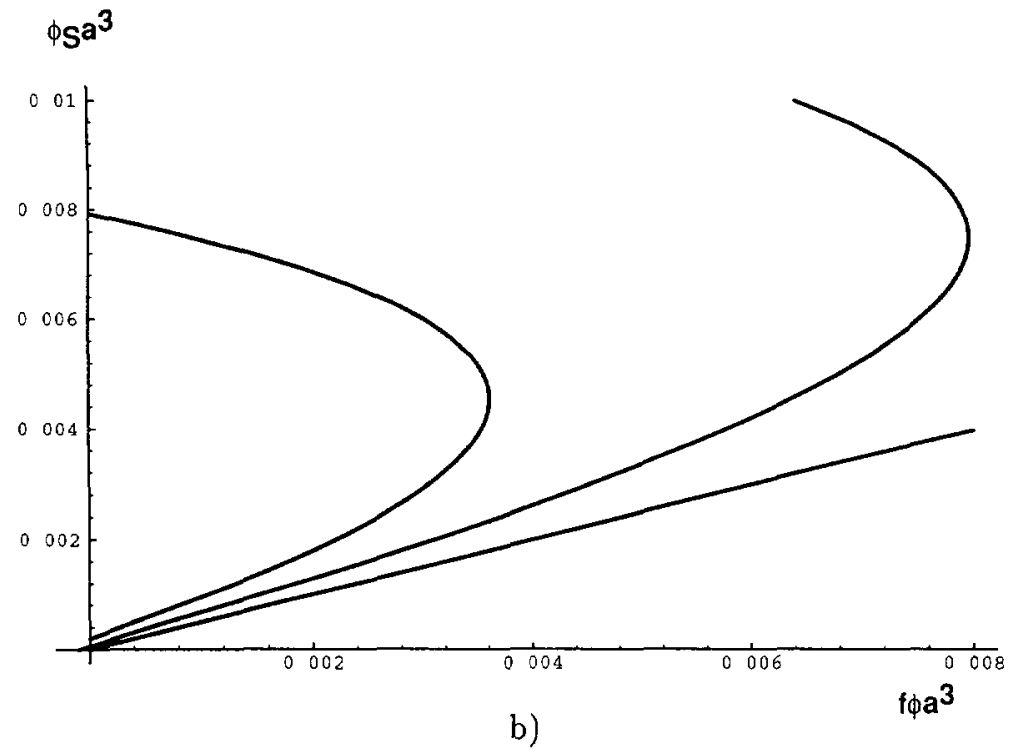

Fig. 1. - a) Line of polymer neutrality in a plane calcium concentration $\phi_{S}$, polymer charge density $x$ for several values of $K_{1} a^{-3}$. From bottom to top, $K_{1} a^{-3}=\infty, 2 \times 10^{4}, 10^{4}$. The monomer size is chosen so that $l_{\mathrm{B}}=2 a$. b) Spinodal line (dashed line) calculated by perturbation around the line of polymer neutrality $\left(K_{1} a^{-3}=10^{4}, l_{\mathrm{B}}=2 a\right)$. The polymer is insoluble between the dashed lines.

We thus first determine the line where the polymer is neutral $S_{\mathrm{e}}=0$, in a second step we look for the spinodal line in the vicinity of this line.

In the limit of weak complexation $K_{1} \prec 24 \pi e^{2} l_{\mathrm{B}}^{3}$ the polymer can never be neutralized and the homogeneous solution is always stable. When $K_{1}$ is larger than this value, the line $S_{\mathrm{e}}=0$ where the polymer is neutralized is given by: 


$$
x=2 \phi_{\mathrm{S}}-\frac{2}{K_{1}} \exp \sqrt{96 \pi l_{\mathrm{B}}^{3} \phi_{\mathrm{S}}}
$$

At a given polymer concentration, the polymer is neutralized by complexation at two salt concentrations: a low salt concentration where the effective complexation constant is large and a high salt concentration where the complexation constant is rather low. The high salt neutralization concentration may be outside the experimental range: for large complexation constants $K_{1} \succ l_{\mathrm{B}}^{3} \exp (\sqrt{96 \pi})$, it is larger than $l_{\mathrm{B}}^{-3}$. In the asymptotic limit where $K_{1} \rightarrow \infty$, the entropic contribution vanishes on the straight line $\phi_{\mathrm{S}}=x / 2$.

At intermediate values of the bare complexation constant, the line $S_{\mathrm{e}}=0$ is a loop closed by the salt axis as shown in Figure 1a. In the vicinity of this line the homogeneous solution is not stable. As only the lower branch of the loop can be observed when the bare complexation constant is large, one or two unstable regions can be found as the salt concentration is increased from zero.

The small parameter $\eta$ defined on the spinodal line via $\phi_{1}=x(1+\eta) / 2$ measures the departure of the spinodal line from the polymer neutralization line $S_{\mathrm{e}}=0$. Neglecting the third virial term in $S$ and assuming that $l_{\mathrm{B}}^{3} \phi_{\mathrm{S}} \ll 1$, we obtain:

$$
\eta= \pm\left[\frac{3 \pi}{2} l_{\mathrm{B}}^{3} \phi_{\mathrm{S}}\right]^{1 / 4}
$$

Combined with the chemical equilibrium law (Eq. (2)), this relation gives an approximate expression for the spinodal lines. The analytical expression is rather tedious, we show in Figure $1 \mathrm{~b}$ an example of spinodal line determined this way. Note however that the $1 / 4$ power in equation (9) can lead in practice to finite corrections even if $l_{\mathrm{B}}^{3} \phi_{\mathrm{S}} \ll 1$.

A more precise determination of the spinodal line requires a numerical solution taking into account all the terms in $S$. The unstable region around the line $S_{\mathrm{e}}=0$, obtained numerically, is shown in Figure 2 in the strong binding limit. Although the line $S_{e}=0$ itself is a straight line in this limit, the unstable region is closed. The upper bound along the polymer axis is due

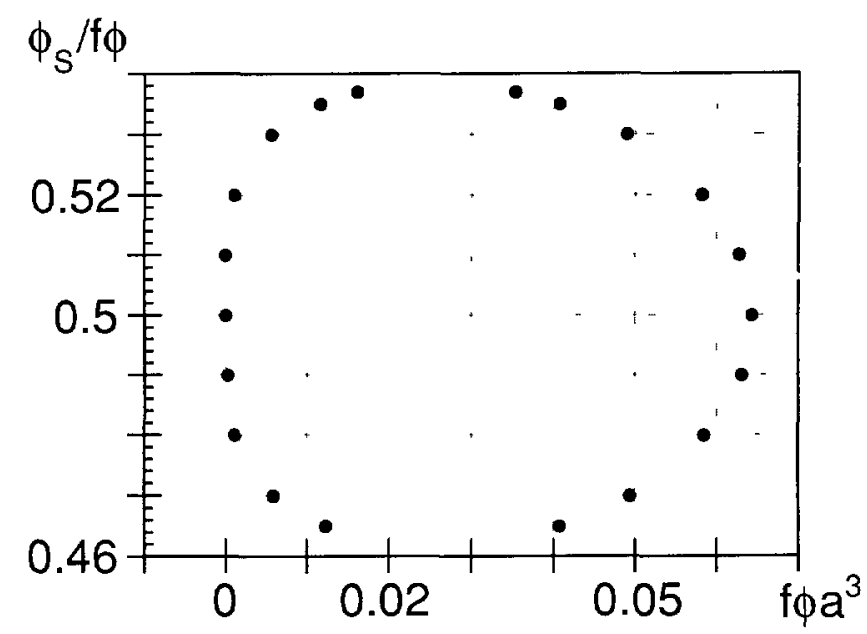

Fig. 2. - Spinodal line for large values of the monocomplexation constant, the parameters are $f=$ $10^{-3}, l_{\mathrm{B}}=2 a, w^{2}=a^{6}$. The vertical axis is the ratio $\phi_{\mathrm{S}} / x$. 


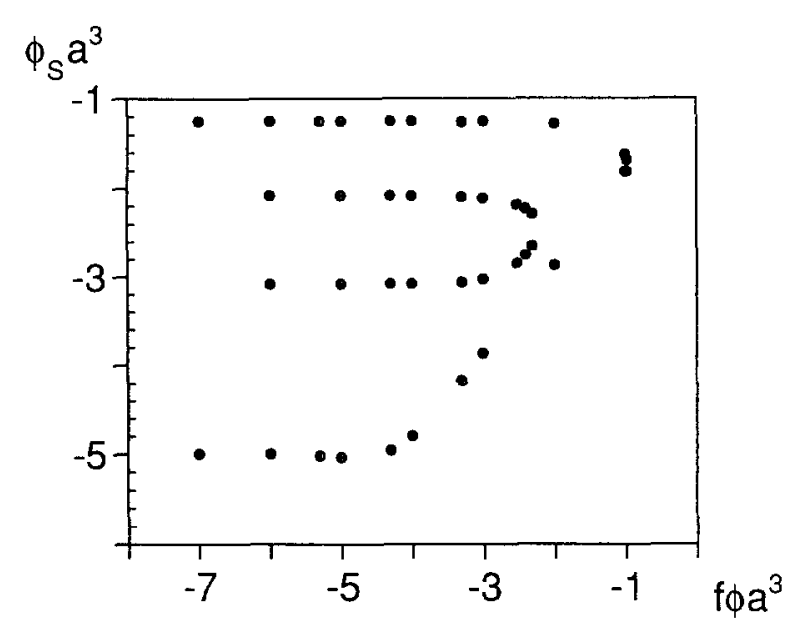

Fig. 3. - Spinodal line in the limit where only dicomplexes form, $K_{2}=10^{9} a^{6}$ (outer line), $K_{2}=10^{6} a^{6}$ (Inner line), $l_{\mathrm{B}}=2 a$.

to the third virial contribution. In the limit where only monocomplexes form, the precipitation of the polymer is thus provoked by an effective neutralization due to the adsorption of calcium ions on the chain and the redissolution is due to charge inversion.

2.4. Formation of Dicomplexes. - By binding onto two charged monomers a calcium ion neutralises two charges of the polyelectrolyte. The polymer is effectively neutral when all the polyelectrolyte charges are bound to calcium ions. This never occurs for entropic reasons. In contrast to the case of monocomplex formation, a fluctuation of the polymer concentration without any loss of entropy of the small ions is thus not possible. The formation of reversible double bonds corresponds to an effective attraction between charged monomers that can dominate over the translational entropy of the small ions.

A simple qualitative equation of the spinodal line at small salt concentrations is obtained as follows. We expand the free energy (Eq. (1)) for $\phi_{2} / x<1$ and obtain an attractive contribution $F_{\text {attr. }} \propto-\phi_{2}$ that provokes the decomposition. At low salt concentrations: $F_{\text {attr. }} \propto$ $-\frac{K_{2} \phi_{\mathrm{S}}}{1+K_{2} x^{2}} x^{2}$ and a negative second virial coefficient of order $-K_{2} \phi_{\mathrm{S}}$ can be defined at small $x$. On the spinodal line this attraction is balanced by the osmotic pressure of the counterions $\Pi=x$, so that $\phi_{2} \propto x$. From the expression of $\mathrm{K}_{2}$ we then obtain the approximate equation of the spinodal line $\phi_{\mathrm{S}} \propto x+\frac{1}{\mathbf{K}_{2} x}$ where all numerical prefactors are omitted. At low salt concentrations $K_{2}$ has the bare value $K_{2}$, the relevant concentration scale is then $1 / \sqrt{K_{2}}$. The salt concentration along the spinodal line decreases with the polymer concentration at low polymer concentrations $\left(x \sqrt{K_{2}}<1\right)$, goes through a minimum at $x \cong 1 / \sqrt{K_{2}}$ and increases linearly for $x>1 / \sqrt{K_{2}}$ with a slope of order one. At higher polymer concentrations, the spinodal lies above this straight line because of the exponential decrease of $\mathbf{K}_{\mathbf{2}}$.

In a marked contrast to the case of monocomplexation the attraction is due to the entropy of mixing of the complexed and non-complexed monomers, given in equation (1). As for the formation of monocomplexes, the non-entropic contributions can be neglected for fairly dilute solutions. Both the destabilizing and the stabilizing effects are entropic effects and the spinodal 
line can be obtained from the analysis of the entropic contribution.

$$
S_{\mathrm{e}}=\left(x-4 \phi_{2}\right)^{2}-12 \phi_{2} \phi_{\mathrm{S}}=0
$$

The explicit form is obtained from the chemical equilibrium law (Eq. (2)) as follows.

The Debye length and therefore the complexation constant $\mathbf{K}_{2}$ only depend on the free calcium density $\phi_{\mathrm{f}}=\phi_{\mathrm{S}}-\phi_{2}$ and on the free counterion density $x$. Inserting $\mathbf{K}_{2}$ in equation (11) gives an explicit expression of $\phi_{2}$ as a function of $x$ and $\phi_{\mathrm{f}}$. For a given polymer concentration, it is then easy to solve (numerically) for the free calcium density $\phi_{\mathrm{f}}$ and thus for $\phi_{\mathrm{S}}$. In Figure 3, typical spinodal lines are shown for different values of $K_{2}$. The spinodal line is a loop closed on the salt axis, going through a minimum at about $x=0.5 / \sqrt{K_{2}}$. When the bare complexation constant is large enough, a linear branch with a slope of about 0.13 is followed by a strong increase. An upper branch at fairly constant salt concentration closes the spinodal on the salt axis. Typical experiments may correspond to the dilute polymer portion of this diagram, with disconnected lower and upper branches.

On the lower branch of the spinodal line, for dilute polymer solutions, $\mathbf{K}_{2}$ is approximately equal to the bare value $K_{2}$. Taking $1 / \sqrt{K_{2}}$ as the concentration unit, the spinodal is determined from

$$
\begin{gathered}
{\left[x \sqrt{K_{2}}-2 \phi_{2} \sqrt{K_{2}}\right]^{2}\left[x \sqrt{K_{2}}-(4-\sqrt{12}) \phi_{2} \sqrt{K_{2}}\right] \times} \\
{\left[x \sqrt{K_{2}}-(4+\sqrt{12}) \phi_{2} \sqrt{K_{2}}\right]-12\left(\phi_{2} \sqrt{K_{2}}\right)^{2}=0}
\end{gathered}
$$

This must be combined with the chemical equilibrium law (Eq. (2)).

For $x \sqrt{K_{2}} \gg 1$ the solution of equation (11) is $\phi_{2}=\frac{x}{4+\sqrt{12}}$. Most calcium ions are then bound to the polymer and

$$
\phi_{\mathrm{S}}=\frac{x}{4+\sqrt{12}} \quad\left(x \sqrt{K_{2}} \gg 1\right)
$$

For vanishing polymer concentration $\phi_{2} \sqrt{K_{2}}=\frac{\left(x \sqrt{K_{2}}\right)^{2}}{\sqrt{12}}+\ldots$ and $\phi_{2} \ll x, \phi_{2} \ll \phi_{\mathrm{S}}$. The chemical equilibrium law gives then:

$$
\phi_{\mathrm{S}}=\frac{1}{\sqrt{12 K_{2}}} \quad(x=0)
$$

Note however that, if the polymer molecular weight is finite, the solution is no longer semidilute in this limit, and our approximations do not remain valid. This result is also valid only if the salt concentration is small enough so that the dependence of the chemical equilibrium constant with the salt concentration can be ignored; we thus require a high enough value of the bare complexation constant $K_{2}$. For too low values of $K_{2}$, there is no precipitation. In the limit $x \sqrt{K_{2}} \prec 1$ there are few complexes formed and:

$$
\phi_{\mathrm{S}} \sqrt{K_{2}}=\frac{1+\left(x \sqrt{K_{2}}\right)^{2}}{\sqrt{12} \sqrt{1+\left(x \sqrt{K_{2}}\right)^{2}}+4 x \sqrt{K_{2}}}
$$

Although this expression is found for $x \sqrt{K_{2}} \prec 1$, it has the correct limit for $x \sqrt{K_{2}} \gg 1$ and gives a fair approximation of the whole lower branch of the spinodal line. The precipitation line given in equation (11) goes through a minimum at $x=0.512 / \sqrt{K_{2}}$ where $\phi_{\mathrm{S}}=0.256 / \sqrt{K_{2}}$ (Fig. 4). 


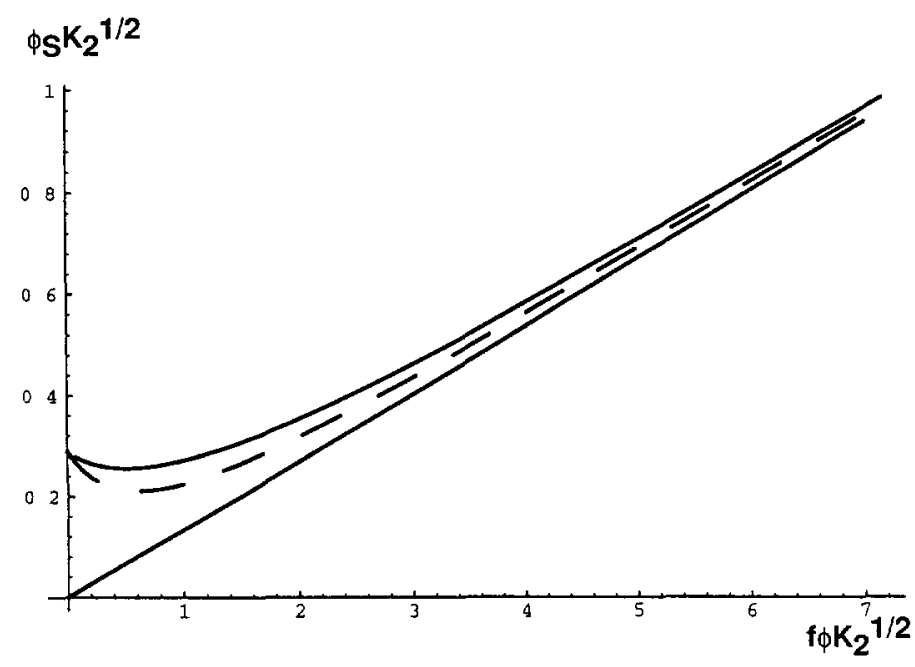

Fig. 4. - Precipitation line at low ionic strength in the case of pure dicomplexation. The concentration unit is chosen as $K_{2}^{-1 / 2}$. The straight line gives the asymptote and the dashed line the approximation of equation (15).

On the upper branch of the spinodal line, the exponential dependence of $\mathbf{K}_{2}$ on ionic strength is important, but there is little calcium bound so that $\phi_{\mathrm{S}}=\frac{1}{\sqrt{12 K_{2}}}$. For dilute polymer concentrations the salt concentration along the upper branch where the polymer becomes soluble again is fairly constant close to that obtained at vanishing polymer concentration:

$$
12 K_{2} \phi_{\mathrm{S}}^{2} \exp \left(-\sqrt{216 \pi l_{\mathrm{B}}^{3} \phi_{\mathrm{S}}}\right)=1
$$

This equation gives both the upper and the lower branches of the spinodal line in the limit where $x \sqrt{K_{2}} \prec 1$. As explained above, there is a threshold for the binding strength: for large enough values of the bare complexation constant, $K_{2} \succ 27\left(\frac{3}{4} \pi l_{\mathrm{B}}^{3} e^{2}\right)^{2}$, equation (15) has two solutions corresponding to the two branches of the spinodal line; for smaller values of the bare complexation constants there is no precipitation. If $K_{2} \succ \frac{l_{\mathrm{B}}^{2}}{12} \exp (\sqrt{216 \pi})$, the salt concentration along the upper branch is outside the experimental range. At intermediate values of the complexation constant, the spinodal line has an upper branch which can be approximated by:

$$
\phi_{\mathrm{S}} l_{\mathrm{B}}^{3}=\frac{\left(\ln \left(12 K_{2}\right)\right)^{2}}{216 \pi}+\frac{4 \ln \left(12 K_{2}\right)}{216 \pi} \ln \left[\frac{\left(\ln \left(12 K_{2}\right)\right)^{2}}{216 \pi}\right]
$$

The low salt branch is given by equation (12).

2.5. Competition Between Mono- And Dicomplexes. - In general, both mono- and dicomplexes form simultaneously, we now discuss their relative importance.

In the limit where mostly monocomplexes form, the polymer precipitation occurs close to the line where the polymer is neutral. Nevertheless, in most cases, the dominant attraction between monomers is due to the formation of dicomplexes and not to the polarization effect. 
In the opposite limit where mostly dicomplexes are formed, the asymptotic behaviour described in the previous section must be recovered. We thus restrict our attention to the entropic term $S_{\mathrm{e}}$ in the spinodal equation that reads:

$$
S_{\mathrm{e}}=\frac{x^{2}-4 \phi_{1} x-8 \phi_{2} x+4 \phi_{1}^{2}-12 \phi_{\mathrm{S}} \phi_{2}+16 \phi_{1} \phi_{2}+16 \phi_{2}^{2}}{2 x^{2} \phi_{1} \phi_{2} \phi_{\mathrm{S}}\left(\phi_{\mathrm{S}}-\phi_{1}-\phi_{2}\right)\left(x-\phi_{1}-2 \phi_{2}\right)}=0
$$

We first discuss the low ionic strength region of the phase diagram and then the region of small polymer concentrations. This allows us to give a classification of all possible phase diagrams.

2.5.1. Low Ionic Strength Limit. - In this section we study the spinodal line at low ionic strength in some detail. In this limit the complexation constants $\mathbf{K}_{1}$ and $\mathbf{K}_{2}$ reduce to the bare values $K_{1}$ and $K_{2}$. It is then convenient to choose $K_{1} / K_{2}$ as the concentration scale and to introduce the dimensionless concentrations $c_{1}=\phi_{i} K_{2} / K_{1}$ (with $c=x K_{2} / K_{1}$ ). The spinodal line is then given by

$$
\begin{gathered}
N(c)=c^{2}\left(1-4 c_{1}-8 c_{1}^{2}\right)-4 c\left(c_{1}+c_{1}^{2}\right)+4 c_{1}^{2}\left(1+3 c_{1}+3 c_{1}^{2}\right) \\
-\frac{K_{2}}{K_{1}^{2}} 12 c_{1}^{2}\left(1+4 c_{1}+4 c_{1}^{2}\right)=0
\end{gathered}
$$

The parameter $p=K_{2} / K_{1}^{2}$ is the equilibrium constant of the process:

2 monocomplexes $\longleftrightarrow 1$ dicomplex +1 free calcium ion

It measures the relative importance of the two complexation processes. The spinodal equation must be solved with the constraint $c \geq c_{1}+2 c_{2}$ which ensures that the total number of complexed monomers remains smaller than the nominal charge of the polymer.

The chemical equilibrium laws (Eq. (2)) impose that on the line $c=c_{1}+2 c_{2}$ where all the monomers form complexes $c=c_{1}$. For the purpose of the discussion it is convenient to consider $N$ given by equation (17) as a function of the polymer concentration $c$, the concentration of monocomplexes $c_{1}$ being kept constant. If there is precipitation and redissolution, $N$ is positive when $c_{1}=c$. The sign of the function $N(c)$ at $c=c_{1}$ therefore indicates the possibility of redissolution by charge inversion.

$$
N\left(c_{1}\right)=\left(c_{1}^{2}+4 c_{1}^{3}+4 c_{1}^{4}\right)(1-12 p)
$$

A detailed analysis of equation (17) along these lines shows that two cases must be considered, depending on the value of $p$ :

(i) when $p<1 / 12$, the spinodal line (Eq. (18)) has two branches at low ionic strength. When increasing the salt concentration, the polymer first precipitates by formation of dicomplexes and is then stabilized by a strong charge inversion due to the monocomplexes.

(ii) when $p>1 / 12$ the spinodal line (Eq. (18)) has only one branch at low ionic strength. Solubilisation by charge inversion is not possible.

(i) $p<1 / 12$, regime of favored monocomplexation

- At low reduced charge concentration $(c \ll 1)$, we find two branches for the spinodal line $c=2 c_{1}(1 \pm \sqrt{3 p})$, and $c_{2} \ll c_{1}$; on each branch, the concentration of free calcium ions $c_{\mathrm{f}}$ goes to a finite value $\frac{p}{1 \pm \sqrt{12 p}}$ so that: 


$$
\phi_{\mathrm{S}}=\frac{x / 2}{1 \pm \sqrt{3 p}}+\frac{p}{1 \pm \sqrt{12 p}} \frac{K_{1}}{K_{2}}
$$

The + sign in equation (19) gives the precipitation line and the - sign, the redissolution line. As $p$ increases, the formation of monocomplexes becomes less dominant and when $p$ approaches $1 / 12$ a diverging amount of free salt is needed to obtain redissolution by charge inversion (note that we are working in the low ionic strength limit).

For vanishing values of $p$, the formation of dicomplexes is a perturbation and the spinodal decomposition occurs close to the line where the polymer is effectively neutral. The electrical polarisation term can then become relevant and the precipitation is described by the mechanism where only monocomplexes form presented in Section 2.3. The direct comparison between the attraction due to the dicomplexes and that due to the polarisation shows that the polarisation terms become dominant when $p=l_{\mathrm{B}}^{2} a / K_{1}$.

- When the polymer concentration is large $(c \gg 1)$, the two branches of the spinodal line correspond to the following limits:

- the lower precipitation line is obtained at a finite value of $c_{1}$ when the coefficient of $c^{2}$ in $N(c)$ vanishes

- the upper redissolution line due to charge inversion is obtained for large values of $c_{1}$.

On the precipitation line, we obtain $c_{1}=\frac{\sqrt{3}-1}{4}, c_{2}=\frac{2-\sqrt{3}}{2} c$ and $c_{\mathrm{f}}=\frac{3+\sqrt{3}}{4} \frac{p}{c}$. Even though $p$ is small, at large polymer concentration, the formation of dicomplexes is dominant and the precipitation line has the same expression as that obtained in Section 2.4:

$$
\phi_{\mathrm{S}}=\frac{1}{4+\sqrt{12}} x+\frac{1}{4(1+\sqrt{3})} \frac{K_{1}}{K_{2}}
$$

The redissolution by charge inversion is obtained when both $c$ and $c_{1}$ are large. Keeping only the leading order terms in $N(c)$, we obtain from equation $(17): c_{1}=\sqrt{\frac{2}{3(1-4 p)}} c$, $c_{2}=\left(1-\sqrt{\frac{2}{3(1-4 p)}}\right) \frac{c}{2}$ and $c_{\mathrm{f}}=\frac{2 \sqrt{2} p}{\sqrt{3(1-4 p)}-\sqrt{2}} c$. The equation of the upper branch of the spinodal line where the polymer redissolves is given by:

$$
\phi_{\mathrm{S}}=\left(\frac{1}{2}+\frac{12 \sqrt{2} p+\sqrt{6-24 p}}{6(1-12 p)}\right) x+f(p) \frac{K_{1}}{K_{2}}
$$

with:

$$
f(p)=\frac{1}{8} \sqrt{\frac{3(1-4 p)}{32}}+\frac{p}{(3 \sqrt{1-4 p}-\sqrt{6}) \sqrt{1-4 p}}+\frac{1}{4 \sqrt{6-24 p}}
$$

At small values $p$ the slope of the solubilization line is about 0.9 , it increases with $p$. At a higher ionic strength, a strong charge inversion ensures the polymer solubility. Finally both the slope and the intercept $f(p)$ in equation (21) diverge at $p=1 / 12$ where only the lower branch (precipitation line) is left.

In Figure 5, the two low ionic strength branches of the spinodal line are plotted in the $\left(c, c_{S}\right)$ plane. Figure 6 shows the complex concentrations along the spinodal line. 


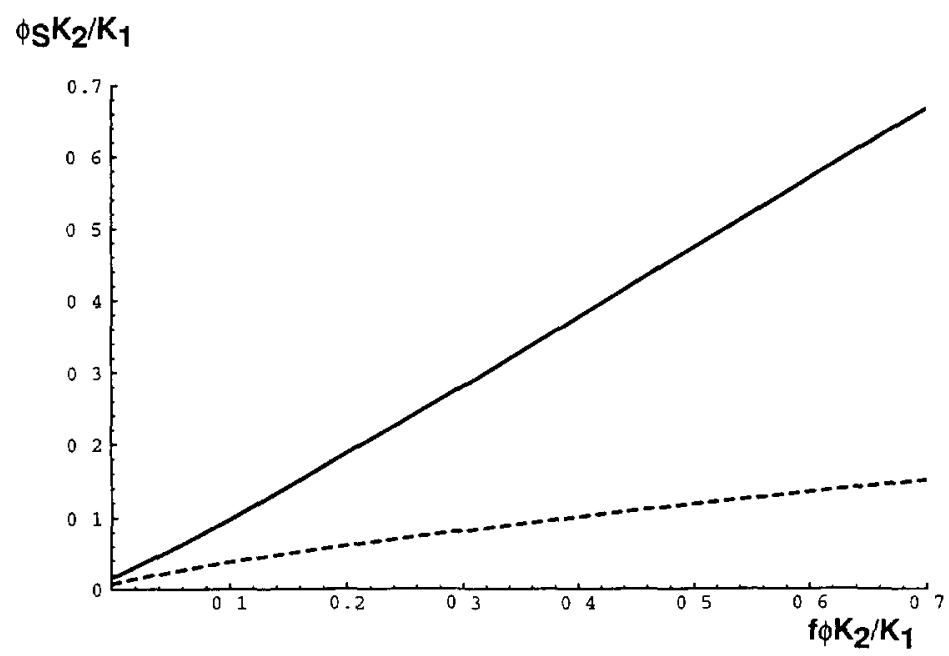

Fig. 5. - Regime of favored monocomplexation $(p<1 / 12)$ : precipitation line and solubilization line at low ionic strength. $(p=0.01)$. The natural concentration unit is $K_{1} / K_{2}$.

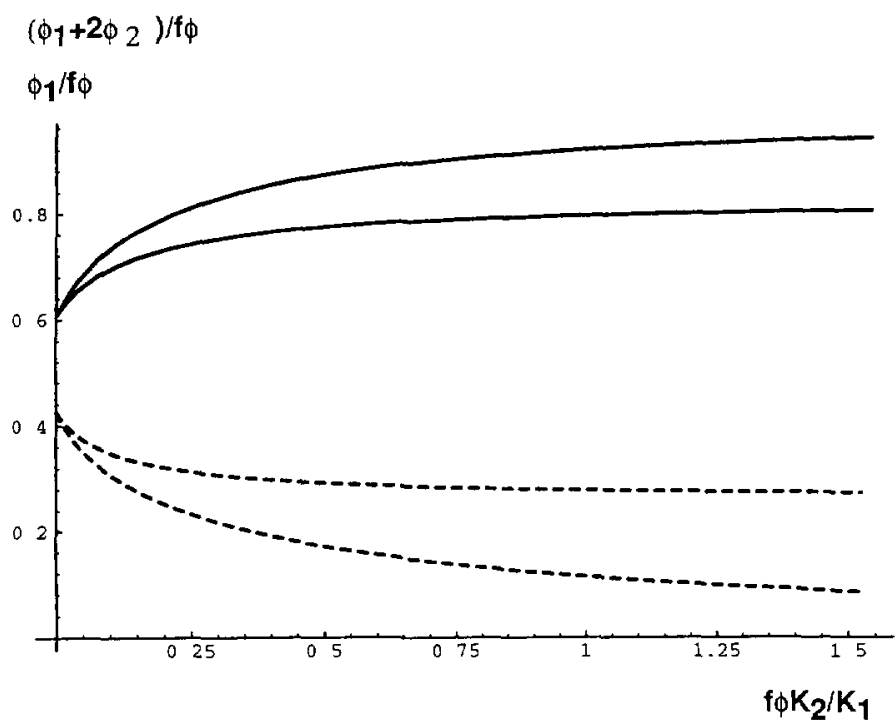

Fig. 6. - Regime of favored monocomplexation $(p<1 / 12)$ : fraction of charged monomers forming monocomplexes $\phi_{1} / x$ and total fraction of monomers forming complexes $\left(\phi_{1}+2 \phi_{2}\right) / x$ along the spinodal line. The lower branches of the plot (dashed lines) correspond to the precipitation line, the upper branches correspond to the solubilization line. $(p=0.01)$.

(ii) $p>1 / 12$, regime of favored dicomplexation

At small ionic strength the spinodal line has only one branch corresponding to the precipitation, solubilisation by charge inversion is not possible. At high reduced polymer concentrations, only dicomplexation is important and the spinodal line is given by the asymptotic form of equation (20). At low polymer concentrations, the formation of monocomplexes dominates over that of dicomplexes and the spinodal line is given by equation (19) with the + sign. 


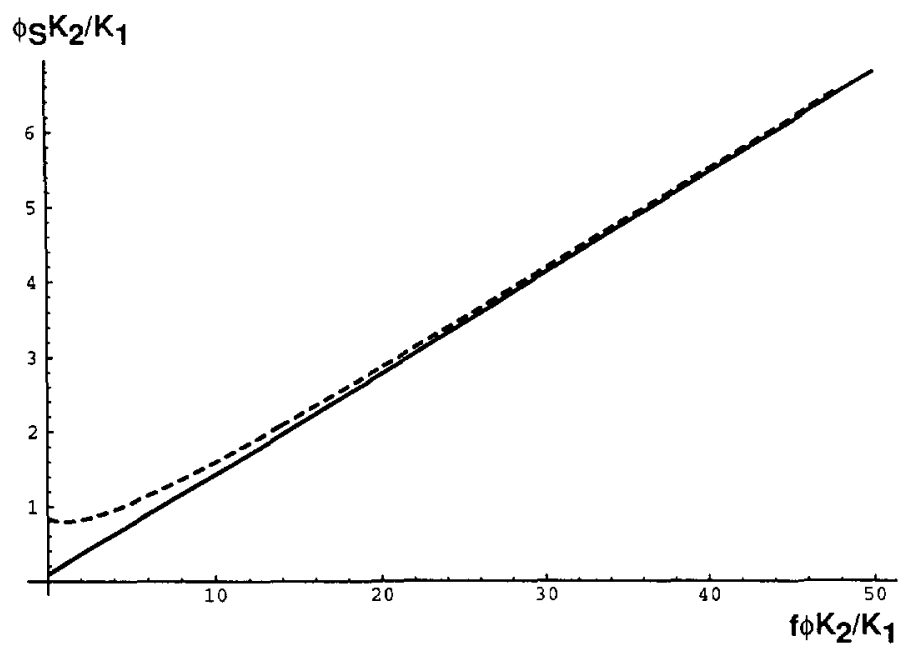

Fig. 7. - Regime of favored dicomplexation $(p>1 / 12)$ : precipitation line at low ionic strength. $(p=10)$.

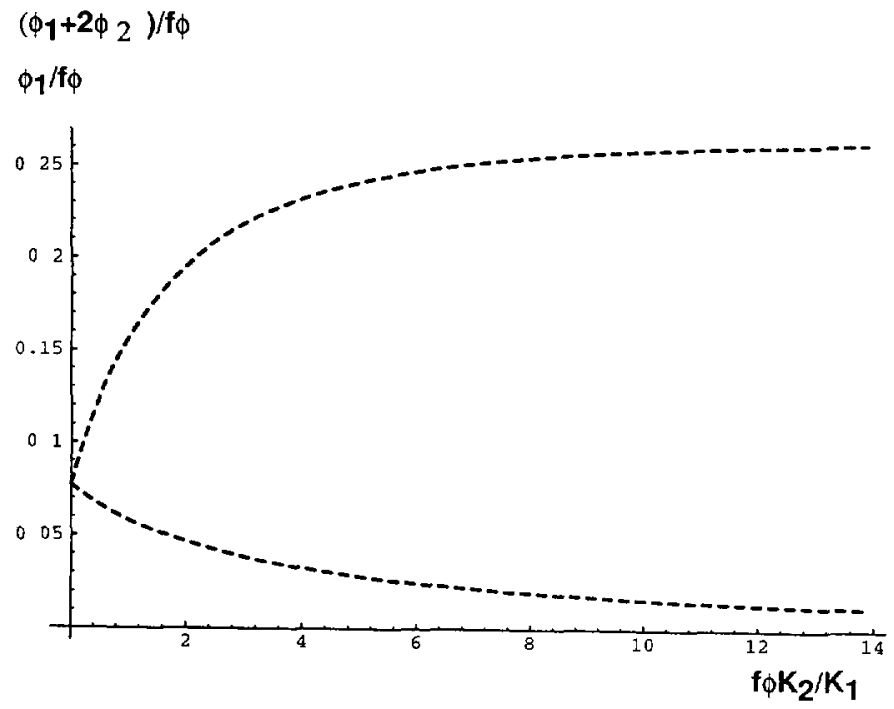

Fig. 8. - Regime of favored dicomplexation $(p>1 / 12)$ : fraction of charged monomers forming monocomplexes $\phi_{1} / x$ and total fraction of monomers forming complexes $\left(\phi_{1}+2 \phi_{2}\right) / x$ along the spinodal line. $(p=10)$.

Figure 7 shows the spinodal line in this limit in the $\left(c, c_{\mathrm{S}}\right)$ plane while Figure 8 shows the complex concentrations along this line.

2.5.2. High Ionic Strength Limit. - In order to get some insight into the high ionic strength part of the phase diagram and to discuss the possible topologies of the phase diagram, it is worth focusing on the limit of vanishing polymer concentrations. We obtain the various topologies of the phase diagram by studying intersections of the spinodal line with the salt 
axis.

Taking into account the variation with the ionic strength, the equilibrium constant between mono and dicomplexes can be written as $\mathbf{p}=\frac{\mathbf{K}_{2}}{\mathbf{K}_{1}}=p \exp \left(\kappa l_{\mathrm{B}}\right)$. For vanishing polymer concentrations the calcium concentration on the spinodal line $c_{\mathrm{S}}$ is given by equation (19) where the bare equilibrium constant $p$ is replaced by $p$. The inverse screening length in this limit is given by $\kappa^{2}=24 \pi l_{\mathrm{B}} \phi_{\mathrm{S}}$ and only depends on $c_{\mathrm{S}}$. The variable $y=\kappa l_{\mathrm{B}}$ on the spinodal line is then the solution of:

$$
g(y)=0, g(y)=\alpha y^{2}-\beta \frac{\exp (2 y)}{1 \pm \sqrt{\beta \exp (y)}}
$$

where $\beta=12 K_{2} / K_{1}^{2}$ and $\alpha=K_{2} /\left(2 \pi l_{\mathrm{B}}^{3} K_{1}\right)$. The two different signs correspond to different branches of the spinodal line. For a given value of $\beta$ this equation only has solutions at high enough values of $\alpha$. The results are summarized in a diagram in the $\left(K_{1}^{2}, K_{2}\right)$ plane shown in Figure 9.

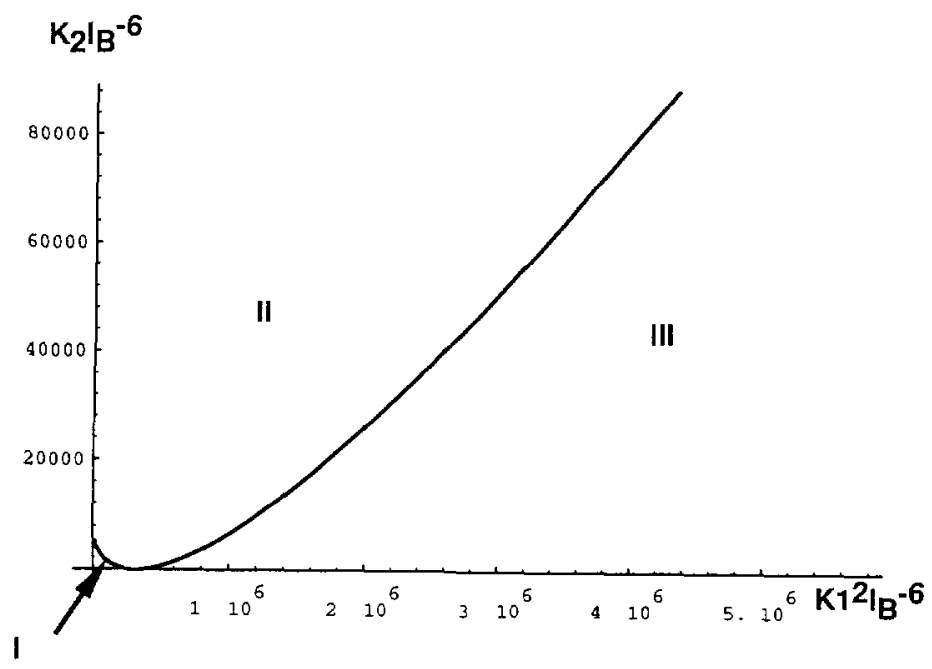

Fig. 9, - Various regimes in the $K_{1}^{2} l_{\mathrm{B}}^{-6}, K_{2} l_{\mathrm{B}}^{-6}$ plane. In regime $I$ the complexation is to weak to provoke separation. In regime II solubilization by charge inversion is not possible. In regime III solubilization by charge inversion is possible.

In regime I, close to the origin, the binding constants are too weak and the homogeneous solution is always stable. In regime II, the spinodal intercepts the salt axis twice as in the case where only dicomplexes form. For a given polymer concentration only one unstable region is found upon increasing the salt concentration. In regime III, there are four intersections of the spinodal line with the salt axis, as in the case where only monocomplexes form, two unstable regions are expected as the salt concentration is increased. When the complexation constants are large, the transition between regime II and regime III occurs at a low ionic strength and is given asymptotically by $K_{2} / K_{1}^{2}=1 / 12$.

For given values of $K_{1}$ and $K_{2}$ it is easy to solve equation (22) numerically and to find the intercepts with the salt axis. Depending on the values of $K_{1}$ and $K_{2}$ the high ionic strength 
branches may not be reached experimentally.

\section{Scattering Function and Mesophase Formation}

Even when the solution is stable against macroscopic concentration fluctuations, it can be unstable against finite size fluctuations. In this case, mesophases with a periodic modulation of the polymer concentration at a finite wave vector form when the salt concentration is increased. Our aim here is to discuss briefly the possibility of mesophase formation prior to the macroscopic decomposition discussed in Section 2.

Mesophase formation has already been predicted for polyelectrolytes in poor solvents. The concentration fluctuations have a characteristic size fixed by a balance between the attraction (here provided by the complexation) and the electrostatic repulsion (or the loss of entropy of the counterions). To characterize the local structure of the solution we compute the polymer/polymer structure factor. This is done at the mean-field level, using the random phase approximation. The system is described by a set of independent concentrations $\left\{\phi_{1}\right\}$. In momentum space the free energy $\delta F$ of a fluctuation at wave vector $q\left\{\delta \phi_{\mathrm{i}}(q)\right\}$ is expanded up to second order in the concentration fluctuations:

$$
\delta F / k T=\frac{1}{2}\left[\chi_{\mathrm{ij}}^{-1} \delta \phi_{1} \delta \phi_{\mathrm{j}}+\frac{4 \pi l_{\mathrm{B}}}{q^{2}} \rho(q) \rho(-q)+S_{0}^{-1}(q) \delta \phi^{2}\right]
$$

The first term on the r.h.s. of equation (23) corresponds to the entropic contributions of the complexes and of the small ions as given by the free energy of equation $(1) \chi_{\mathrm{ij}}^{-1}=$ $\frac{\partial^{2}(F / k T)}{\partial \phi_{\mathrm{i}} \partial \phi_{\mathrm{j}}}$. The second term is the electrostatic energy of the charge fluctuation $\rho(q)$ associated with the concentration fluctuation $\left\{\delta \phi_{1}(q)\right\}$. The last term corresponds to the polymer chain concentration fluctuation where $S_{0}$ is the structure factor of a Gaussian polymer chain given by the so-called Debye function. The partition function is obtained by integration of $\exp (-\delta F / k T)$ over the concentration fluctuations. We define an effective polymer partition function $Z_{\mathrm{p}}(\delta \phi(q))=\exp \left(-\delta F_{\mathrm{p}} k T\right)$ by tracing over all the fluctuating concentrations except the polymer concentration $\delta \phi(q)$. The effective polymer free energy can then be written as $\delta F_{\mathrm{p}}=\delta \phi(q) S^{-1}(q) \delta \phi(-q) / 2$ where $S(q)$ is the polymer-polymer structure factor:

$$
S^{-1}(q)=S_{0}^{-1}(q)+f^{2} \frac{4 \pi l_{\mathrm{B}} s-2 \phi_{2} q^{2}}{x\left(\left(x+2 \phi_{2}\right) q^{2}+4 \pi l_{\mathrm{B}}\left(-s-4 \phi_{1} x-6 \phi_{2} x+2 x^{2}+6 \phi_{\mathrm{S}} x\right)\right)}
$$

with

$$
s=4 \phi_{1}^{2}-12 \phi_{\mathrm{s}} \phi_{2}+16 \phi_{1} \phi_{2}+16 \phi_{2}^{2}-4 \phi_{1} x-8 \phi_{2} x+x^{2}
$$

and

$$
S_{0}^{-1}(q)=a^{2} q^{2} / 12 \phi
$$

For convenience we only use here the large wave vector approximation of the Debye function. The line $s=0$ corresponds to the spinodal line given in equations $(16,18)$. In the absence of complexation $\left(\phi_{1}=\phi_{2}=0\right)$ the second term in equation (24) reduces to the electrostatic interaction screened by the small ions $\left(\mathrm{Na}^{+}, \mathrm{Ca}^{2+}, \mathrm{Cl}^{-}\right)$.

We do not want to give here a full description of mesophase formation but we only want to discuss the possibility of mesophase formation in the vicinity of the spinodal line where the solution becomes unstable with respect to macroscopic fluctuations. In the region of the phase 


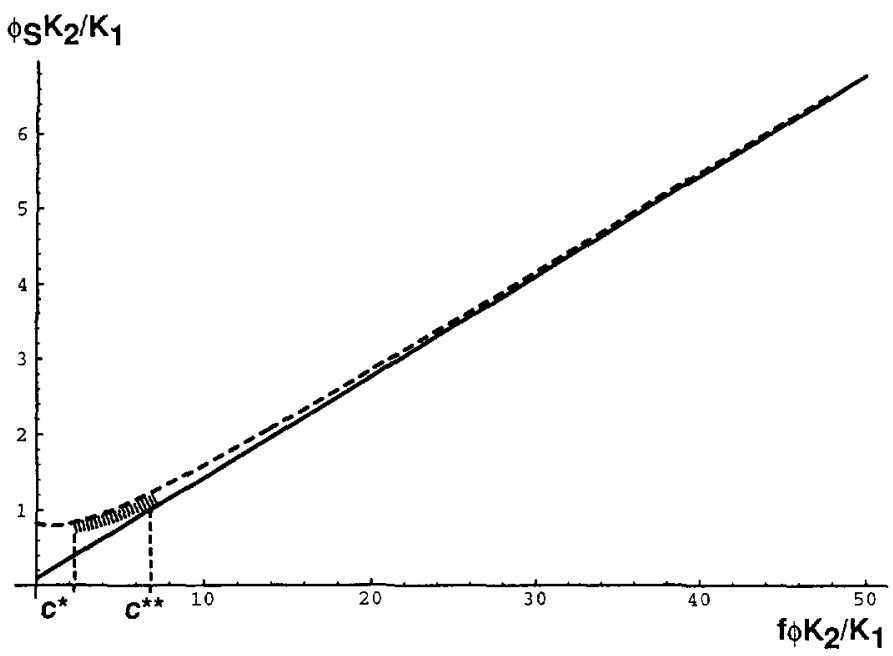

Fig. 10. - Region of instability at a finite wave vector along the macroscopic spinodal line: mesophases are predicted in the concentration range $\left[c^{*}, c^{* *}\right]$ where $c=f \phi K_{2} / K_{1}$.

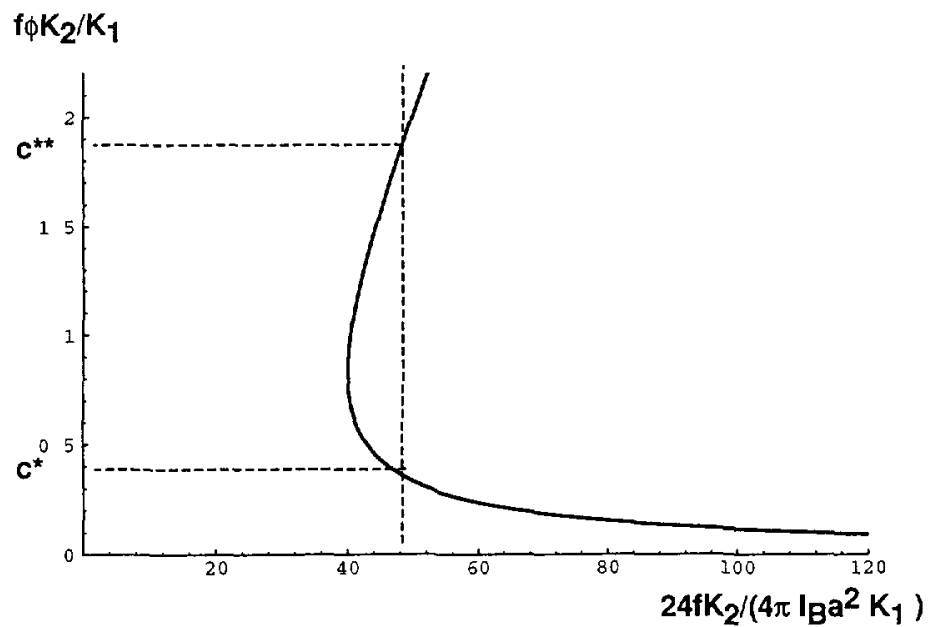

Fig. 11. - Variation of the concentrations where mesophases form on the spinodal line as a function of the parameter $24 f K_{2} / 4 \pi l_{\mathrm{B}} a^{2} K_{1}$. Mesophase separation takes place prior to macro-separation concentrations inside the curve. The curve corresponds to $p=1$.

diagram where the solution is macroscopically stable but close to being unstable $(s>0)$, the inverse structure factor equation (24) formally vanishes for two values of the square of the wave vector $q^{2}$. However these two formal solutions correspond to an instablity towards mesophase formation only if the corresponding values of $q^{2}$ are positive. One easily checks that this is not the case at very small or very large values of the polymer concentration on the spinodal line of macroscopic phase separation. The first instability undergone by the solution is thus a macrophase separation (precipitation). However along this spinodal line in an intermediate range of concentration $\left(c^{*}<c<c^{* *}\right)$, the inverse structure factor vanishes for two finite 
wave vectors indicating that mesophase formation can occur at smaller salt concentrations than the macroscopic precipitation of the solution. This is qualitatively shown in Figure 10. The variation of the two concentrations $c^{*}$ and $c^{* *}$ with the parameter $24 f K_{2} /\left(4 \pi l_{\mathrm{B}} a^{2} K_{1}\right)$, is displayed in Figure 11 for $p=1$.

\section{Conclusion}

We have obtained phase diagrams for polyelectrolyte solutions in the presence of a divalent salt. The calculated phase diagrams compare well with the experimental phase diagrams of the so-called $L$-type [5-7,10], where small calcium salt concentrations are sufficient to induce the precipitation of a polyelectrolyte solution. Two mechanisms have been considered to account for the ion polyelectrolyte interaction:

- monocomplexation where the adsorbed calcium ion locally inverts the charge and turns the polyelectrolyte into a polyampholyte

- dicomplexation where the adsorbed calcium ion bridges between two charged monomers.

The formation of monocomplexes reduces the net charge of the polymer and thus the coupling between the polymer and the counterions. In the absence of dicomplexation, the polymer precipitates when it is almost neutral. The precipitation is due to a polarisation effect very similar to that proposed for polyampholytes. A small excess of divalent salt inverses the net charge of the polymer provoking a redissolution. When the equilibrium constant for monocomplexation is large, all the calcium ions bind to the polyelectrolyte and the precipitation occurs for a calcium concentration $\phi_{S}$ equal to half the polymer charge concentration $f \phi$. This result may be extended to the case of a $z$-valent salt where we expect $\phi_{\mathrm{S}}=f \phi / z$.

The reversible formation of bridges by dicomplexes corresponds to an effective attraction between charged monomers. This attraction causes a precipitation of the polyeletrolyte when it is larger than the stabilizing pressure of the counterions. At high salt concentrations the binding equilibrium constants of the calcium ions decrease. This promotes ionic dissociation and thus an increase in the concentration of free calcium ions. In our model, the desorption of the calcium ions explains the redissolution of the polyelectrolyte at a high ionic strength.

Monocomplexation is favored against dicomplexation when the equilibrium constant associated with the chemical equilibrium:

\section{2 monocomplexes $\longleftrightarrow 1$ dicomplex +1 free calcium ion}

is smaller than a threshold value (1/12 in the strong complexation limit in our model). Even when monocomplexes are favored, the precipitation is due to the formation of dicomplexes; a strong charge inversion due to the monocomplexes causes the redissolution. At high calcium concentration we predict a second precipitation also followed by redissolution if the complexation is not too strong.

When the formation of dicomplexes is favored, we find a single precipitation followed by a redissolution at a high salt concentration, that is fairly independent of the polymer concentration. In both regimes, monocomplexation dominates at low polymer concentrations.

Throughout the paper we have discussed polymer chains in the limit of infinite molecular weight. The extension of our results to finite molecular weights is straightforward. The "translational" entropy of the chains eliminates the precipitation at very low polymer concentrations. 
The spinodal line as shown for example in Figure 3 is then a closed loop. The point on the left of the curve where the polymer concentration is minimum occurs however at very low polymer concentrations and in most of the diagram one can consider the molecular weight as infinite.

Our model is crude and remains at the mean-field level. It does not apply to strong polyelectrolytes for which Manning condensation [11,12] certainly plays an important role. Manning condensation is close to a strong monocomplexation: however, in the experiments performed with polystyrene sulfonate where Manning condensation is expected to play an important role, the salt concentration on the precipitation line does not correspond to an almost neutralized polyelectrolyte [7]. A specific, purely electrostatic theory has been proposed recently [15].

The discussion of polyelectrolyte complex formation in a mixture of oppositely charged polyelectrolytes in the weak coupling limit by Borue and Erukhimovitch is closer to our approach. Their model accounts for both electrostatic and short range monomer/monomer interactions [16].

The formations of both monocomplexes and dicomplexes are described here by phenomenological ion-polymer interaction parameters which enter the equilibrium constants. We thus propose a two-parameter theory. Nevertheless our predictions are either independent of the parameters (in some specific limits) or can be described by master curves; this should provide a strong test of the theory.

The macroscopic precipitation of the solution is not the only phase transition that can occur in these systems, as recently proposed for polyelectrolyte solutions in a poor solvent, a microscopic phase separation can lead to the formation of mesophases where the polymer concentration is modulated at a finite period. We have checked that there is no mesophase separation prior to the macrophase separation at low charge fractions or, at higher charge fractions, at low polymer concentrations. A detailed study of the mesophases for fairly high charge fractions in an intermediate concentration range has not yet been done but would be worthwhile. In this context, a recent variational calculation of the mesophases in polyelectrolyte systems by Nyrkova et al. seems to be of a special interest [17].

\section{Acknowledgments}

We thank L. Belloni, M. Delsanti, M. Drifford and M. Olvera de la Cruz (CEN Saclay), J. François (I.C.S Strasbourg), L. Leibler (E.S.P.C.I. Paris), I. Nyrkova and A. Semenov (Moscow) and F. Tanaka (Tokyo) for useful discussions and comments.

\section{References}

[1] des Cloizeaux J and Jannink G., Les polymères en solution (Les Editions de Physique, Les Ulis, 1987).

[2] Pfeuty P., Velasco R. and de Gennes P.G., J Phys. France Lett. 38L (1977) 5; Pfeuty P., J. Phys. France 39 (1978) C2-149.

[3] Khokhlov A., J. Phys. A 13 (1980) 979.

[4] Borue V. and Erukhimovitch I., Macromolec 21 (1988) 3240; Joanny J.F. and Leibler L,. J. Phys. France 51 (1990) 545.

[5] Axelos M., Mestdagh M. and François J., Macromolec., in press (1994).

[6] Tuong N.D., Solin J.C., François J. and Pham Q.T., Polym. Commun. 25 (1984) 208. 
[7] Delsanti M., Dalbiez J P., Spalla O., Belloni L. and Drifford M., Macro Anion Characterization: From Dilute Solutions to Complex Fluıd, K.S. Schmitz, Ed. (A.C.S. Book, 1994).

[8] Schaad P., Thomann J.M., Voegel J.C. and Gramain P., Collovds Surf. A 83 (1994) 285.

[9] Ikegani A. and Imai N., J Polym. Scr. 56 (1962) 133.

[10] Tuong N.D. and François J., Solid Liquid Interaction in Porous Media, Vol. 42 (Ed. Technic, Paris, 1985) p. 251.

[11] Manning G.S., J Chem. Phys. 51 (1969) 924.

[12] Manning G.S., Rev. Brophys. 11 (1978) 179.

[13] Leibler L and Pezron E., Space Time Organization in Macromolecular Fluids, F. Tanaka, M. Doi and T. Ohta, Eds. (Springer, Berlin, 1989); Pezron E., Leibler L. and Lafuma F., Macromolec. 22 (1989) 2656.

[14] Tanaka F. and Matsuyama A., Phys. Rev. Lett. 62 (1989) 2759

[15] Olvera de La Cruz M., Belloni L., Delsanti M., Dalbiez J.P., Spalla O. and Drifford M., "Precipitation of Highly Charged Polyelectrolyte Solutions in Presence of Multivalent Salts", submitted to J Chem. Phys.; Bellonı L., Olvera de La Cruz M., Delsanti M., Dalbiez J.P., Spalla O. and Drifford M., "Polyelectrolyte Solutions + Multivalent Salts = Phase Separation", First International Conference on Scaling Concepts in Complex Fluids, Catanzaro, Italy, July 1994, to appear in Nuovo Cim.

[16] Borue V. and Erukhımovitch I., Macromolec. 23 (1990) 3625.

[17] Nyrkova I.A, Khokhlov A.R. and Doi M., Macromolec 27 (1994) 4220. 